\title{
Low temperature photoluminescence properties of Zn-doped GaAs
}

\author{
M.K. Hudait ${ }^{\mathrm{a}, \mathrm{b}, *}$, P. Modak ${ }^{\mathrm{b}}$, K.S.R.K. Rao ${ }^{\mathrm{c}}$, S.B. Krupanidhi ${ }^{\text {a }}$ \\ a Materials Research Centre, Indian Institute of Science, Bangalore 560 012, India \\ ${ }^{\mathrm{b}}$ Central Research Laboratory, Bharat Electronics, Bangalore 560 013, India \\ ${ }^{\mathrm{c}}$ Department of Physics, Indian Institute of Science, Bangalore 560 012, India
}

Received 25 June 1998

\begin{abstract}
Dimethylzinc (DMZn) was used as a p-type dopant in GaAs grown by low pressure metalorganic chemical vapor deposition (MOCVD). The influence of growth parameters, such as, DMZn mole fractions, growth temperature, trimethylgallium (TMGa) mole fractions, substrate surfaces on the $\mathrm{Zn}$ incorporation have been studied. The surface morphology of the layers was measured by scanning electron microscopy (SEM). The hole concentrations and zinc (Zn) incorporation efficiency are studied by using Hall effect, electrochemical capacitance voltage (ECV) profiler, and low temperature photoluminescence (LTPL) spectroscopy as functions of hole concentration $\left(10^{17}-1.5 \times 10^{20} \mathrm{~cm}^{-3}\right)$ and experimental temperatures $(4.2-300 \mathrm{~K})$. The hole concentration increases with increasing DMZn and TMGa mole fractions and decreases linearly with increasing growth temperature. The main PL peak shifted to lower energy and the full width at half maximum (FWHM) increased with increasing hole concentration. An empirical relation for FWHM, $\Delta E \mathrm{p}$, band gap, $E \mathrm{~g}$, and band gap shrinkage, $\Delta E \mathrm{~g}$ in $\mathrm{Zn}$ doped GaAs as a function of hole concentration were obtained. These relations are considered a useful tool to determine the hole concentration in $\mathrm{Zn}$ doped GaAs by low temperature PL measurement. The hole concentration increases with increasing TMGa mole fraction and the main peak is shifted to lower energy side. (C) 1998 Elsevier Science S.A. All rights reserved.
\end{abstract}

Keywords: Gallium arsenide; Epitaxy; Optical properties; Semiconductors

\section{Introduction}

Undoped and Zn-doped GaAs epitaxial layers grown by low pressure metalorganic chemical vapor deposition (MOCVD) [1] are widely used in optoelectronic device fabrications [2]. In addition to high optical and electrical quality of the films, the heavily doped epitaxial film for optoelectronic device applications require mirror smooth surface morphology. The mirror smooth surface morphology and specific concentration of impurity in GaAs by MOCVD can be obtained by controlling the growth temperature. Hence, it is essential to understand the influence of the growth parameters, which control the layer quality.

The effect of p-type heavy doping $\left(>10^{19} \mathrm{~cm}^{-3}\right)$ in GaAs is an important issue of the optical and electrical properties not only in the view of a fundamental understanding but also for the device applications. The heavy doping affects the density of states, carrier mobility,

* Corresponding author. Tel.: + 91-80-3311330; Fax: + 910-803341683; e-mail: sbk@mrc.iisc.ernet.in. absorption, band structures, luminescence properties, and hence the device performance [3,4]. Also, the high doping affects the band gap shrinkage and by a band tail extending into the gap. In the heterojunction-based devices, the band gap shifts due to heavy doping result in valence and conduction band discontinuity of the heterojunction interface [5].

The p-type doping in GaAs can be obtained either of $\mathrm{C}, \mathrm{Be}, \mathrm{Zn}$, or $\mathrm{Mg}$ as doping sources. $\mathrm{Zn}$ is the most common dopant in GaAs and AlGaAs, although the diffusion coefficient of $\mathrm{Zn}$ in these III-V compound semiconductors is high [6], and it is preferred because $\mathrm{Zn}$ precursors can be handled very easily and high doping efficiency $\left(10^{16}-10^{20} \mathrm{~cm}^{-3}\right)$, uniform doping can be obtained. Zn doping is always exhibiting low memory effects, a relatively small amount of deep level traps [7] and good surface morphology even at high doping levels. S. Z. Sun et al [8] found that the surface morphology of Zn-doped GaAs was more dependent on growth rate than growth temperature when the mole fraction of diethylzinc (DEZn) was $9.16 \times 10^{-5}$. Because of the different electrical and optical properties of 
Zn doped GaAs from C-doped GaAs [9-13] grown by MOCVD, these properties have been studied extensively by several authors in recent years [14-19]. This prompted us to conduct a systematic study of $\mathrm{Zn}$ doping in GaAs by low temperature photoluminescence (PL) measurement.

Low temperature photoluminescence (LTPL) spectroscopy is a common characterization technique for investigating the band structures of GaAs at high doping levels. Band gap shrinkage due to heavy doping is a well known phenomenon in III-V compound semiconductors, particularly observed in GaAs by PL spectroscopy $[9,10,20-26]$. The luminescence properties are dependent on the growth conditions (methods), doping concentrations, impurity species, growth temperatures, etc. In this paper we are reporting the results from the low temperature luminescence properties of $\mathrm{Zn}$-doped GaAs by PL measurement by varying the hole concentrations, trimethylgallium (TMGa) mole fractions and PL measurement temperatures. After thorough investigation of Zn-doped GaAs, we have suggested a relationship for full width at half maximum (FWHM), band gap and band gap shrinkage of Zn-doped GaAs, which could be considered a useful tool for determining the hole concentration in Zn-doped GaAs by low temperature PL measurement.

\section{Experimental}

The $\mathrm{Zn}$-doped p-type GaAs were grown in a low pressure horizontal MOCVD reactor on both Cr-doped semi-insulating and Si-doped $\mathrm{n}^{+}$-GaAs (100) substrates with an offset by $2^{\circ}$ towards [110] direction. The source materials were trimethylgallium (TMGa), arsine $\left(\mathrm{AsH}_{3}\right)$, dimethylzinc (DMZn) as a p-type dopant and palladium purified $\mathrm{H}_{2}$ as a carrier gas. During the growth, the pressure inside the reactor was kept at 100 Torr and the growth temperature was varied from 550 to $725^{\circ} \mathrm{C}$. $\mathrm{AsH}_{3}$ flow rate was kept constant at 50 standard $\mathrm{cm}^{3} \mathrm{~min}^{-1}$ (SCCM) and the TMGa flow rate was varied from 10 to 20 SCCM in order to obtain different growth rates and same stoichiometry. The total flow rate was about 2 standard liter $\mathrm{min}^{-1}$ (SLPM). The growth rate was varied between 470 and $800 \AA \mathrm{min}^{-1}$. The details of the growth procedure can be found elsewhere [27].

The surface morphology of GaAs epilayers was seen under scanning electron microscopy (SEM). The doping concentrations were determined by using both BioRad electrochemical capacitance-voltage (ECV) polaron profiler and Hall measurement. Hall effect by Van der Pauw method was carried out at temperature $300 \mathrm{~K}$ for mobility measurement, considering the Hall factor $r_{H}=1$. The hole concentration does not change appreciably within the temperature range of $77-300 \mathrm{~K}$.
The hole concentration measured at $300 \mathrm{~K}$ was used in 4.2 and $120 \mathrm{~K}$ photoluminescence studies and the same procedure was followed by several authors $[14,18,25,28]$. P-type layers with thickness of about 1.2 $\mu \mathrm{m}$ were chosen for analysis to reduce thickness measurement errors. Hole densities in the range of $10^{17}$ $1.5 \times 10^{20} \mathrm{~cm}^{-3}$ were measured.

PL measurements were carried out using a MIDAC Fourier Transform PL (FTPL) system at temperatures ranging from 4.2 to $300 \mathrm{~K}$ and $100 \mathrm{~mW}$ laser power. Argon ion laser operating at a wavelength of $5145 \AA$ was used as a source of excitation. The exposed area was about $3 \mathrm{~mm}^{2}$. PL signal was detected by a $\mathrm{LN}_{2}$ cooled Ge Photodetector whose operating range is about $0.75-1.9 \mathrm{eV}$, while resolution was kept at 0.5 $\mathrm{meV}$.

\section{Results and discussion}

$\mathrm{Zn}$ was used as a p-type dopant for GaAs and it is the most common MOCVD p-type dopant for GaAs and AlGaAs. All the Zn-doped GaAs epitaxial layers were grown for this study at a V/III ratio of 87 [29].

\subsection{Electrical properties}

\subsubsection{Effect of variation of DMZn mole fraction and growth temperature}

The surface of epilayer is influenced by the growth parameters, such as, pressure inside the reactor tube, growth temperature, substrate orientation, $\mathrm{AsH}_{3} /$ TMGa mole ratio, etc. The surface of $\mathrm{Zn}$-doped p-type GaAs appears to be mirror smooth over a wide range

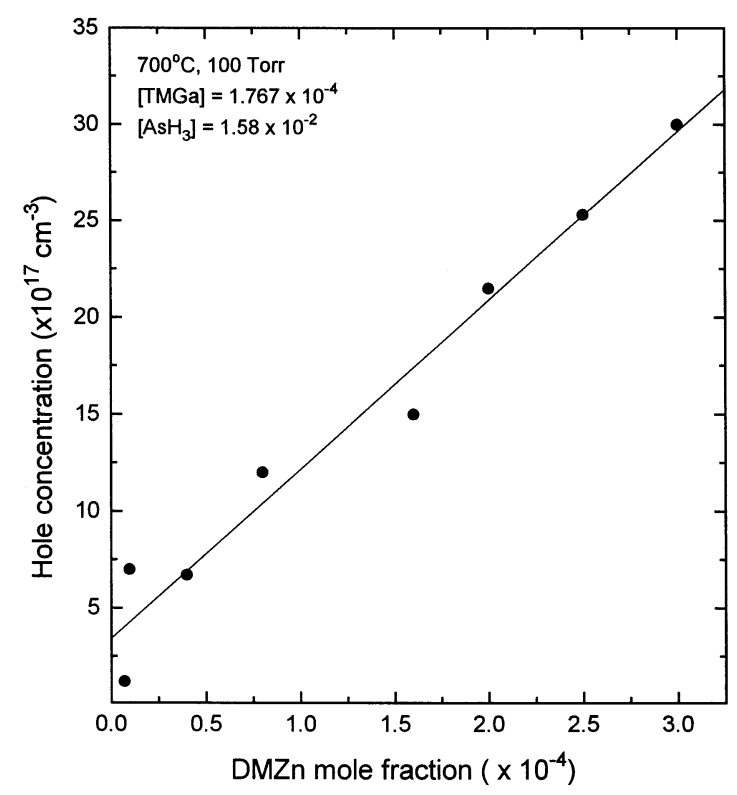

Fig. 1. Hole concentration of zinc doped GaAs as a function of DMZn mole fraction. 


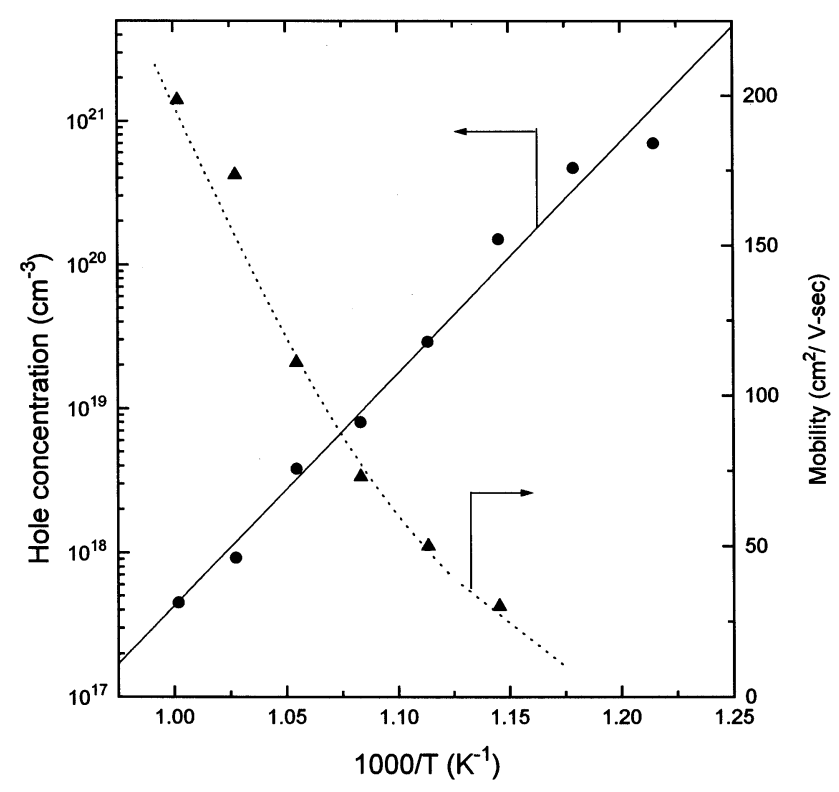

Fig. 2. $300 \mathrm{~K}$ hole carrier concentrations and hole mobilities for $\mathrm{Zn}$-doped GaAs epilayers grown under various growth temperatures. (M. F. of $\mathrm{TMGa}=1.767 \times 10^{-4}, \mathrm{M}$. F. of $\mathrm{AsH}_{3}=1.58 \times 10^{-2}, \mathrm{M}$. F. of $\mathrm{DMZn}=6.68 \times 10^{-5}$ ).

of temperatures $\left(600-725^{\circ} \mathrm{C}\right)$ in this study. Fig. 1 shows the hole concentration as a function of DMZn mole fraction.

The hole concentration increases linearly with DMZn mole function, which is consistent to that described by Glew [30]. The hole concentration was observed to increase as the growth temperature decreases as shown in Fig. 2 for a fixed DMZn mole fraction. The strong temperature dependence of $\mathrm{Zn}$ incorporation was believed to be a result of evaporation of $\mathrm{Zn}$ atoms from the growth surface due to increased vapor pressure at higher temperatures. Between 600 and $725^{\circ} \mathrm{C}$ the incorporation activation energy of DMZn from the slope of the Arrhenius plot is around $3.2 \mathrm{eV}$, which is in agreement with the earlier reported results by Bass and Oliver [31] and Glew [30]. The surface morphology of $\mathrm{Zn}$-doped layers was found polycrystalline in the range of temperature 550 to $575^{\circ} \mathrm{C}$ and can be seen from the Fig. 3. From this figure we observed that the surface morphology of $\mathrm{Zn}$-doped GaAs not only depend on growth temperature but also on the type of substrate surfaces (semi-insulating or $\mathrm{n}^{+} \mathrm{GaAs}$ substrate) of the same growth run. TMGa decomposes rapidly above $500^{\circ} \mathrm{C}$ in an MOCVD reactor. Between 500 and $550^{\circ} \mathrm{C}$ cracked $\mathrm{AsH}_{3}$ concentration is normally low. This has been suggested due to the lack of cracked $\mathrm{AsH}_{3}$ concentration on the growth surface [2]. Above $575^{\circ} \mathrm{C}$, the concentration of arsenic is significant [2] and plays a major role in the growth kinetics. Hole concentrations of $\mathrm{Zn}$ doped GaAs films are known to depend upon $\mathrm{AsH}_{3}$ partial pressures. Therefore, $\mathrm{Zn}$ incorporation changes with temperature and decomposed $\mathrm{AsH}_{3}$ concentration. Above $600^{\circ} \mathrm{C}, \mathrm{AsH}_{3}$ was fully decomposed and $\mathrm{Zn}$ incorporation was temperature dependent. For a given TMGa, DMZn, and $\mathrm{AsH}_{3}$ mole fraction, the hole concentration and Hall mobility are also seen in Fig. 2. The hole mobility exhibited a decrease with decreasing growth temperature. This phenomena can be interpreted in terms of pronounced $\mathrm{Zn}$ evaporation from the surface of epilayers at higher growth temperature. Another mechanism that may explain these results is temperature dependence of gallium vacancies. Chang et al [16] and Glew [30] observed similar behavior of $\mathrm{Zn}$ doped GaAs by low pressure MOCVD reactor using DEZ, and both DMZn and DEZ, respectively.

A vacancy-controlled model may be considered to explain such behavior. In the $\mathrm{TMGa}-\mathrm{AsH}_{3}$ system, the leading reaction to the formation of GaAs can be expressed as:

$\left(\mathrm{CH}_{3}\right)_{3}+3 / 2 \mathrm{H}_{2}+\mathrm{V}_{\mathrm{Ga}} \stackrel{K_{1}}{\Leftrightarrow} \mathrm{Ga}_{\mathrm{Ga}}+3 \mathrm{CH}_{4}$

$\mathrm{AsH}_{3}+\mathrm{V}_{\mathrm{Ga}} \stackrel{K_{2}}{\Leftrightarrow} \mathrm{As}_{\mathrm{As}}+3 / 2 \mathrm{H}_{2}$

Where $K_{1}$ and $K_{2}$ are the equilibrium constants of the above reactions, then

$\frac{\left[V_{\mathrm{Ga}}\right]}{\left[V_{\mathrm{As}}\right]} \equiv \frac{K_{2}}{K_{1}} \frac{P_{\mathrm{AsH}_{3}}}{P_{\mathrm{TMGa}}} \frac{P_{\mathrm{CH}_{4}}^{3}}{P_{\mathrm{H}_{2}}^{3}}$.

Here $V_{\mathrm{Ga}}$ and $V_{\mathrm{As}}$ represent the gallium and arsenic vacancies, respectively. $\mathrm{Ga}_{\mathrm{Ga}}$ represents the gallium occupying on gallium site and $\mathrm{As}_{\mathrm{As}}$, the arsenic occupying on arsenic site. An increase in $P_{\mathrm{AsH}_{3}} / P_{\mathrm{TMG} a}$ will increase in gallium vacancy concentration, hence, the incorporation of $\mathrm{Zn}$ on $\mathrm{Ga}$ site increased. The hole concentration thus increased when the $\mathrm{AsH}_{3}$ mole fraction is increased [32].

According to analysis of Vechten $[33,34]$ the vacancy migration reaction occurring in GaAs growth can be expressed as:

$\mathrm{As}_{\mathrm{As}}+\mathrm{V}_{\mathrm{Ga}} \Leftrightarrow \mathrm{As}_{\mathrm{Ga}}+\mathrm{V}_{\mathrm{As}}$

where $\mathrm{As}_{\mathrm{Ga}}$ is the arsenic occupying on Ga site.

Here As must obtain enough energy to overcome the energy barrier. When the growth temperature decreases, the above reaction occurs towards the left and more gallium vacancies tend to appear. Since the incorporation of $\mathrm{Zn}$ is proportional to $\mathrm{Ga}$ vacancy, the $\mathrm{Zn}$ concentration on $\mathrm{Ga}$ sites increases at lower growth temperatures.

\subsubsection{Effect of growth temperature on growth rate}

The change in growth rate as a function of growth temperature for $\mathrm{Zn}$ doped GaAs as shown in Fig. 4. It can be seen from this figure that the growth rate increases with increasing growth temperature up to $625^{\circ} \mathrm{C}$. But different authors observed [30] different 

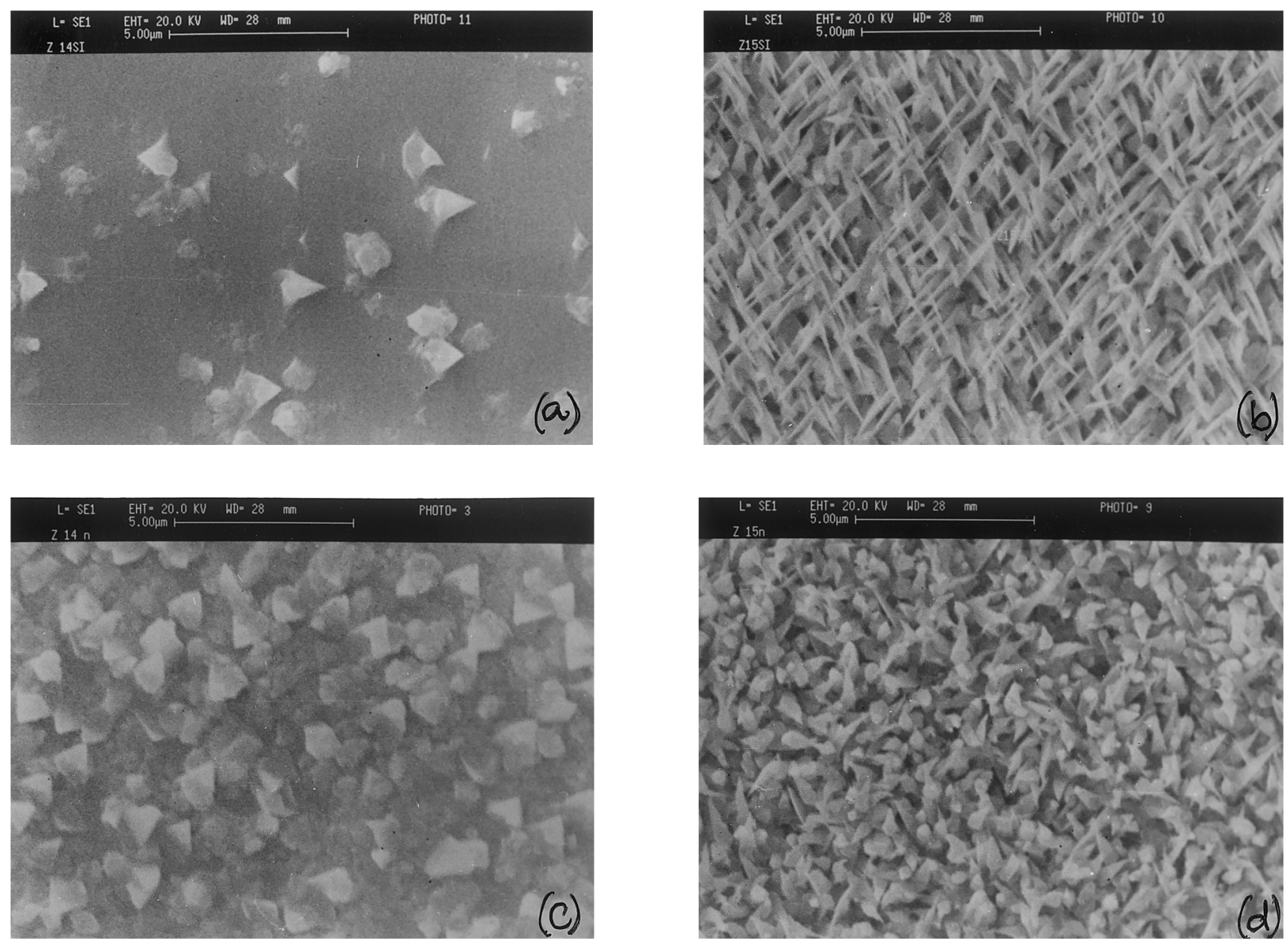

Fig. 3. SEM micrograhs of (a) and (b) at $575^{\circ} \mathrm{C}, 550^{\circ} \mathrm{C}$ on semi-insulting GaAs and (c) and (d) at $575^{\circ} \mathrm{C}, 550^{\circ} \mathrm{C}$ on semiconducting GaAs substrates, respectively.

behavior of growth rate in the temperature range 550 $750^{\circ} \mathrm{C}$. At high substrate temperatures, the growth rate decreases because of arsenic desorption effects from the growth surface. Between the temperatures 625 and $725^{\circ} \mathrm{C}$ the growth rate was found to be temperature independent which is similar to that described by Glew [30]. In this temperature region, TMGa is fully decomposed and hence growth rate becomes independent of temperature. In a classic paper, Shaw [35] showed that an examination of growth rate versus temperature allows a general categorization of the process, limiting the growth rate as either mass transport, surface kinetics or thermodynamics. For an exothermic process, such as MOCVD, increasing temperature results in a decrease in the thermodynamically limited growth rate. If the reaction-rate-limits the growth rate, which is termed the kinetically limited case, the growth rate increases with increasing temperature. Since diffusion is a nearly temperature independent process, the growth rate is nearly independent of substrate temperature in the mass-transport-limited case, as also observed by Shaw [35]. The growth rate is nearly independent of temperature of MOCVD growth process in the temperature range from 550 to $750^{\circ} \mathrm{C}$, at normal operating pressure. This is indicative of mass-transport-limited growth. At lower temperatures, the growth rate decreases with decreasing temperature which happens to be characteristic of a process-limited by reaction kinetics. The growth rate is proportional to the flux of atoms being transported, usually by diffusion, through the gas phase to the interface, which is identical to the flux of atoms crossing the interface into the solid. Using ordinary growth conditions, keeping temperature between $\approx 550$ and $800^{\circ} \mathrm{C}$, the growth rate is nearly independent of temperature in the mass-transport-limited growth. For surface-kinetically-limited process, the growth rate increases exponentially with increasing temperature. This occurs for the MOCVD growth of GaAs only at temperature below $\approx 550^{\circ} \mathrm{C}$. Since the MOCVD growth process is in the mass-transport limited regime, the growth rate is linearly dependent on the group III partial pressures not the group $\mathrm{V}$ partial pressure, which controls the stoichiometry of the semiconductor. This is important because the stoichiometry affects the 
dopant and impurity incorporation as well as the concentrations of native defects, i.e. those involving interstitial atoms and vacancies. It is observed that higher the substrate temperature $\left(T_{\text {sub }}\right)$, higher the available gallium concentration and hence higher growth rates.

\subsection{Optical properties}

\subsubsection{PL spectra as a function of hole concentrations}

Fig. 5 (a), (b), and (c) shows the 4.2, 120, and $300 \mathrm{~K}$ PL spectra of $\mathrm{Zn}$-doped GaAs for hole concentrations of (a) $4.5 \times 10^{17} \mathrm{~cm}^{-3}$, (b) $3.8 \times 10^{18} \mathrm{~cm}^{-3}$, (c) $8 \times 10^{18}$ $\mathrm{cm}^{-3}$, (d) $2.9 \times 10^{19} \mathrm{~cm}^{-3}$, and (e) $1.5 \times 10^{20} \mathrm{~cm}^{-3}$, respectively. The curves were intentionally offset along $y$-axis with respect to each other for better clarity. The same procedure was used for all the other PL spectra in this paper. The FWHM, $\Delta E(\mathrm{p})$ of the donor-to-acceptor (e-A) peak between the temperature 4.2 and $300 \mathrm{~K}$ of PL spectra increases with increasing hole concentration. The broadening of FWHM is similar to the C-doped GaAs [28], Be-doped GaAs [24] and Zn-doped GaAs [9] and can be explained by band-to-band optical transitions with and without momentum (K) conservation between the conduction and valence bands [9]. Alternatively, this can be explained as the impurity band merges with the valence band edge and it becomes band tail states at high doping concentrations. Because of this phenomena, the optical transitions between the conduction and valence band are broadened, the FWHM of PL spectra increases. The $\Delta E(\mathrm{p})$ increases slowly up to $\mathrm{p} \cong 1 \times 10^{18}$ $\mathrm{cm}^{-3}$ and increases rapidly with increasing hole concen-

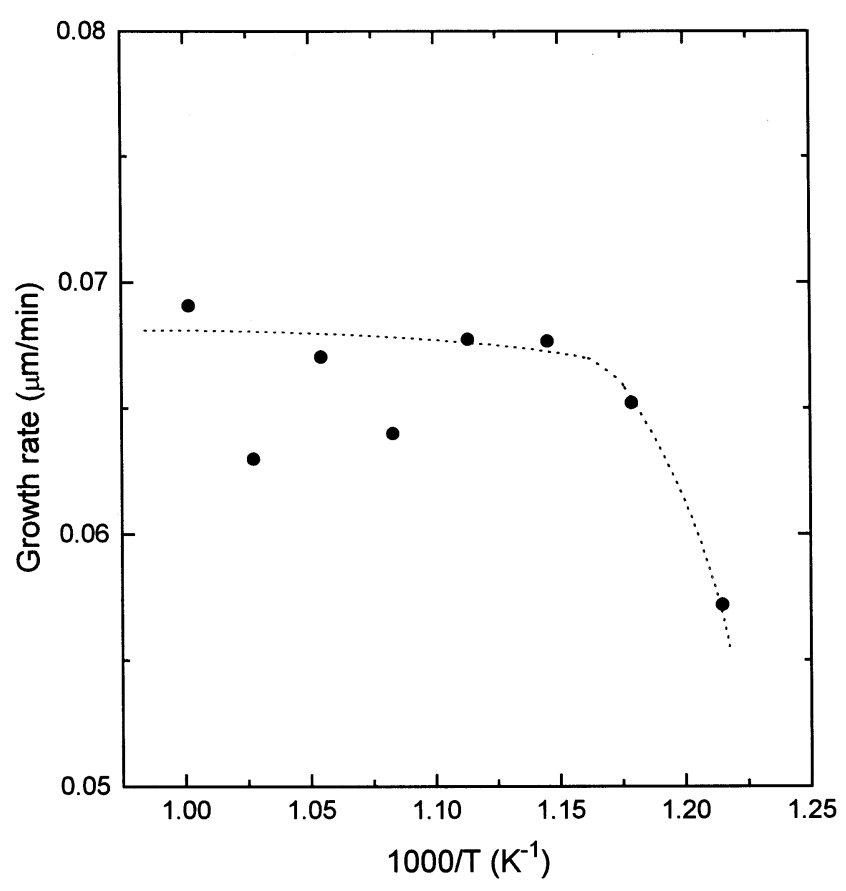

Fig. 4. Growth rate versus reciprocal of growth temperature. tration. From the data we have obtained an empirical relation for FWHM of $\mathrm{Zn}$ doped GaAs at $4.2 \mathrm{~K}$,

$\Delta E(p)(\mathrm{eV})=1.15 \times 10^{-8} p^{1 / 3}$

with the concentration range between $1 \times 10^{17}$ and $1.5 \times$ $10^{20} \mathrm{~cm}^{-3}$. The results are compared with C-doped GaAs [10] and shown in Fig. 6. The value of FWHM is about $10 \mathrm{meV}$ for $p=1 \times 10^{18} \mathrm{~cm}^{-3}$, and it increases rapidly at $p>3 \times 10^{18} \mathrm{~cm}^{-3}$ due to the appearance of the extra peak which is clearly observed at $4.2 \mathrm{~K} \mathrm{PL}$ measurement temperature. When the concentration reaches $1.5 \times 10^{20} \mathrm{~cm}^{-3}$, the value of FWHM is $50 \mathrm{meV}$.

\subsubsection{Band gap shrinkage due to doping effect}

The main peak energy shifted to lower energy as the hole concentration increased, which is primarily because of doping induced band gap shrinkage or band gap narrowing (BGN). Fig. 7 shows the main PL peak energy, $E_{\mathrm{p}}$ with the hole concentration in the range of $1 \times 10^{17}-1.5 \times 10^{20} \mathrm{~cm}^{-3}$. The $E_{\mathrm{p}}$-p relation is very important for the determination of hole density in the base of heterojunction bipolar transistors (HBTs) and active layer of the laser diodes. The empirical relation for $E_{\mathrm{p}}-\mathrm{p}$ at $4.2 \mathrm{~K}$ by PL measurement can be represented by

$E_{\mathrm{p}}=1.52-4.1 \times 10^{-6} \times p^{0.215}$

We have also included the peak energy measured at 120 and $300 \mathrm{~K}$ by PL measurement. From this graph, it is seen that the peak energy measured at 4.2 and $120 \mathrm{~K}$ are the same and hence the $E_{\mathrm{p}}$-p relation can be used to considered a useful tool for determination of hole concentration at any temperature within 4.2 and $120 \mathrm{~K}$. If this is the case, one should expect the same band gap narrowing (BGN) due to heavy doping in GaAs by low temperature PL measurement at 4.2 and $120 \mathrm{~K}$ It is also seen from this figure that the peak energy measured at $300 \mathrm{~K}$ by PL measurement is lower compared to 4.2 and $120 \mathrm{~K}$. For a fixed hole density, the peak energy shifted to lower energy with increasing PL measurement temperature can be seen from Fig. 8. It is very difficult to extract the exact band gap shift from the PL spectra because of life time broadening effects [36]. We determined the band gap, $E_{\mathrm{g}}$ (Fig. 9) of heavily doped GaAs, by a linear extrapolation to the energy axis, using a function of the type $\mathrm{f}(E)=\mathrm{A}\left(E-E_{\mathrm{g}}\right)^{1 / 2}$, of the spectrum to the background level following the work by Olego and Cardona [9]. This method was used by several authors for determination of $E_{\mathrm{g}}$.

Fig. 10 (a) shows the band gap shrinkage of Zn-doped GaAs in the range of $4.5 \times 10^{17}-1.5 \times 10^{20} \mathrm{~cm}^{-3}$, as a function of hole concentration. In this figure, we have also plotted the reported results for $\mathrm{Zn}, \mathrm{Be}$, and $\mathrm{C}$ doped GaAs measured at temperatures between 4.2 and $77 \mathrm{~K}$, as the band gap shrinkage is independent of temperature $[9,20]$. The measured band gap shrinkage of carrier concentration of $\mathrm{Be}, \mathrm{C}$, and 

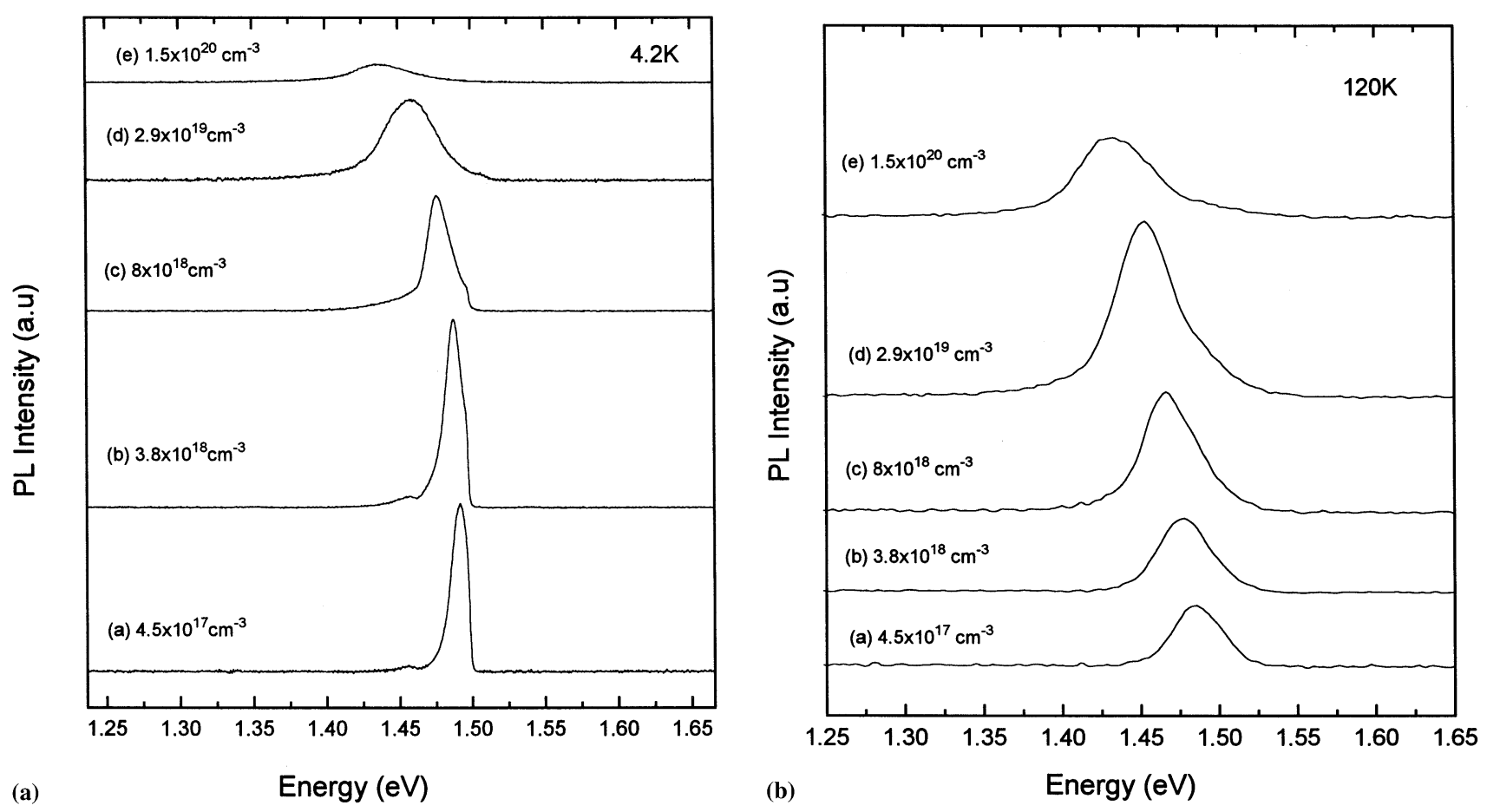

(a)
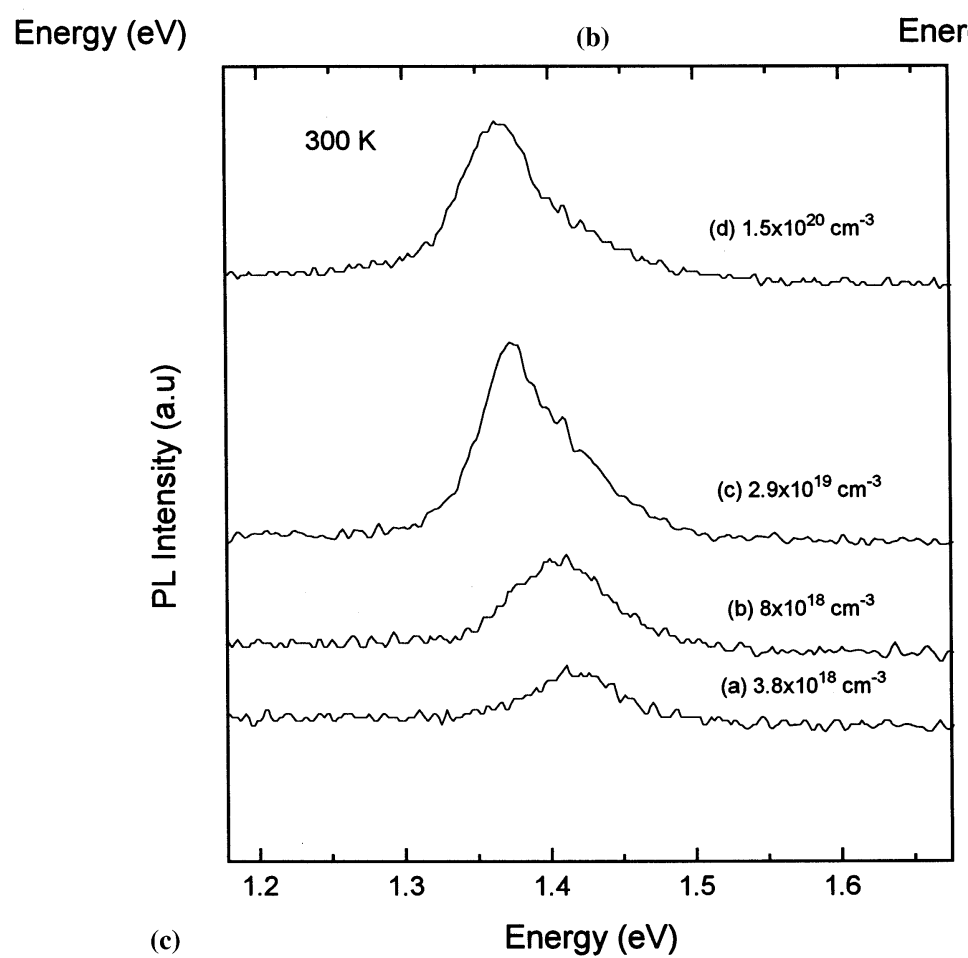

Fig. 5. PL spectra of Zn-doped GaAs epilayers for various hole concentrations (a) $4.2 \mathrm{~K}$, (b) $120 \mathrm{~K}$, (c) $300 \mathrm{~K}$.

Zn-doped GaAs, exhibited consistent agreement between each other $[9,14,18,24,25,28,37]$. Fig. 10 (b) shows the band gap shrinkage of our results at the temperature ranges from 4.2 to $300 \mathrm{~K}$. In general, the band gap shrinkage is proportional to the hole concentration of the form $\mathrm{p}^{1 / 3}$, thus it can be represented by

$$
\Delta E_{\mathrm{g}}=E_{\mathrm{g}}(\text { doped })-E_{\mathrm{g}}(\text { pure })=-B \mathrm{p}^{1 / 3}
$$

where $\mathrm{B}$ has been adjusted to give the measured value of $E_{\mathrm{g}}$ at higher hole concentrations and the minus sign signifies the band gap shrinkage at higher concentrations. The empirical relation for band gap narrowing with our data at $4.2 \mathrm{~K}$ can be written as

$\Delta E_{\mathrm{g}}=-2.75 \times 10^{-8} \mathrm{p}^{1 / 3}$

where $\Delta E_{\mathrm{g}}$ is in $\mathrm{eV}$ and $\mathrm{p}$ in $\mathrm{cm}^{-3}$. 


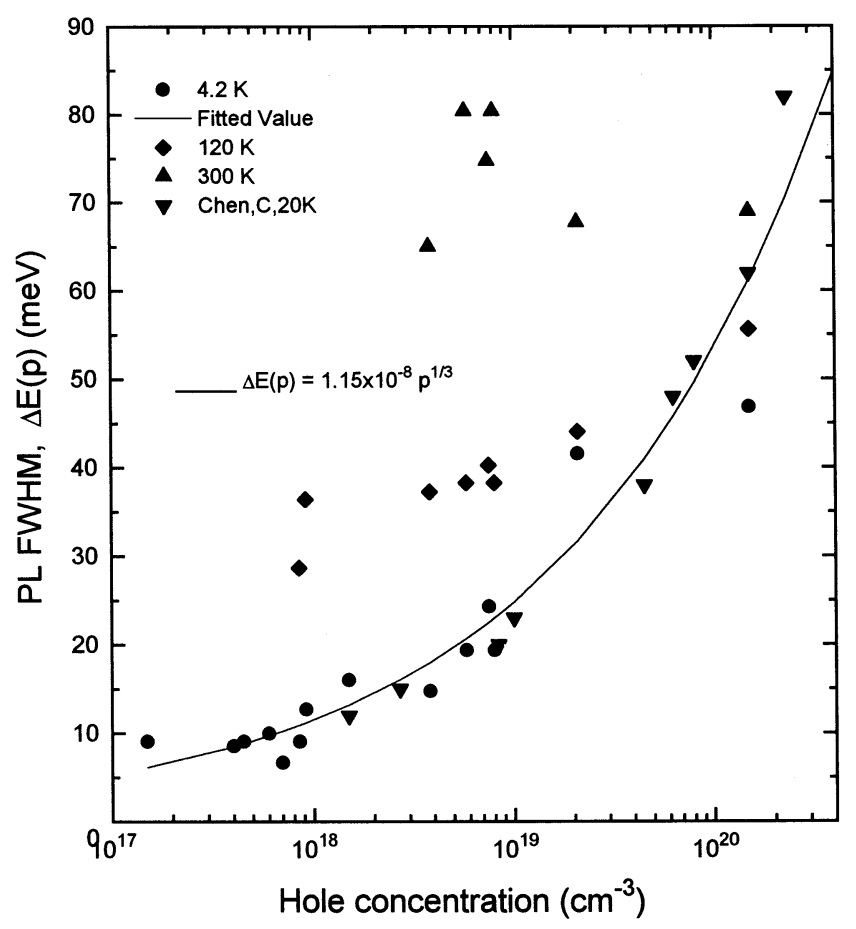

Fig. 6. FWHM of PL spectra at different temperatures versus hole concentrations.

We have made an attempt to fit our experimental data to the expression suggested by S.C. Jain [38],

$E_{\mathrm{g}}=E_{\mathrm{g}}(0)-\Delta E_{\mathrm{g}}(p)$

Where $\Delta E_{\mathrm{g}}(\mathrm{p})=\mathrm{a} \times \mathrm{p}^{1 / 3}+\mathrm{b} \times \mathrm{p}^{1 / 4}+\mathrm{c} \times \mathrm{p}^{1 / 2}, \mathrm{a}, \mathrm{b}$, and

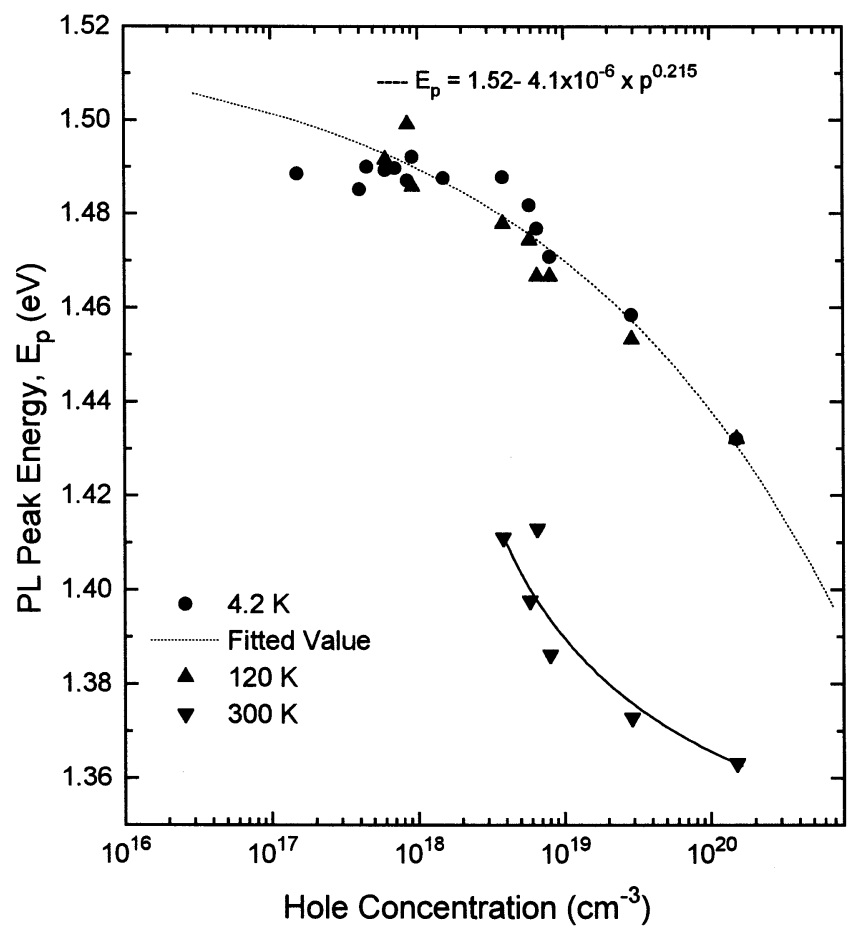

Fig. 7. PL peak energy $E_{\mathrm{p}}$ as a function of hole concentration.

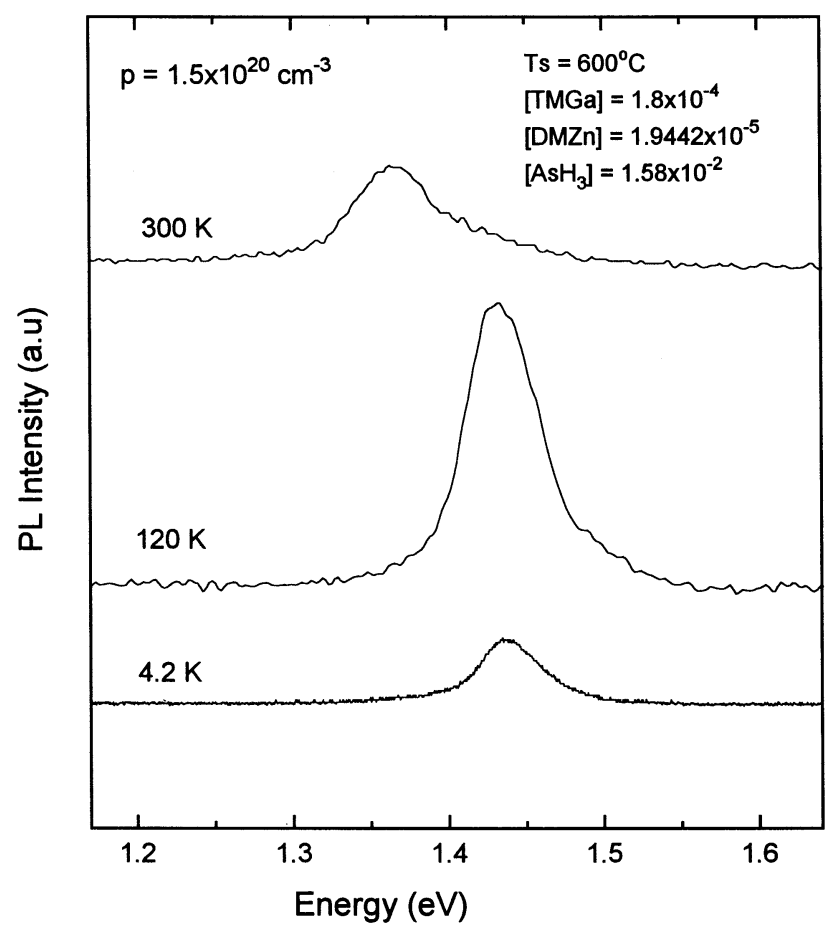

Fig. 8. PL spectra as a function of temperature for a specific hole concentration.

c are the coefficients that represent the effects of the BGN due to majority-majority carrier exchange, minority-majority correlation and carrier-ion interaction, respectively. For p-type GaAs, the constants a, b, and c are $9.83 \times 10^{-9}, 3.9 \times 10^{-7}$, and $3.9 \times 10^{-12}$, respec-

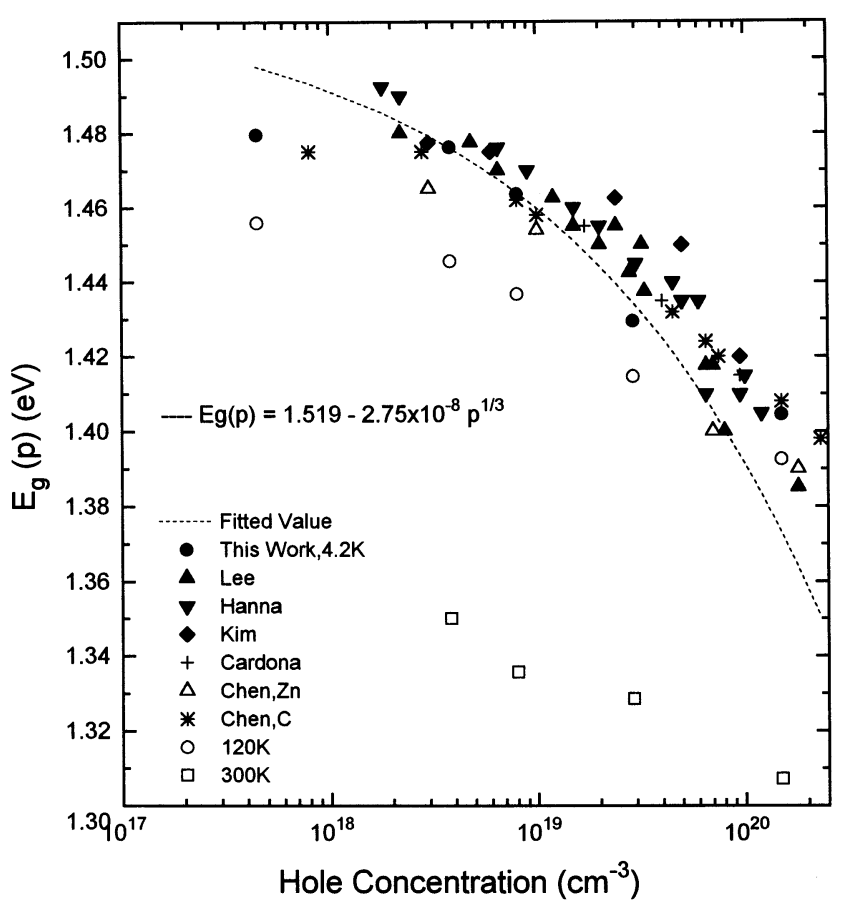

Fig. 9. Band gap versus hole concentration. 

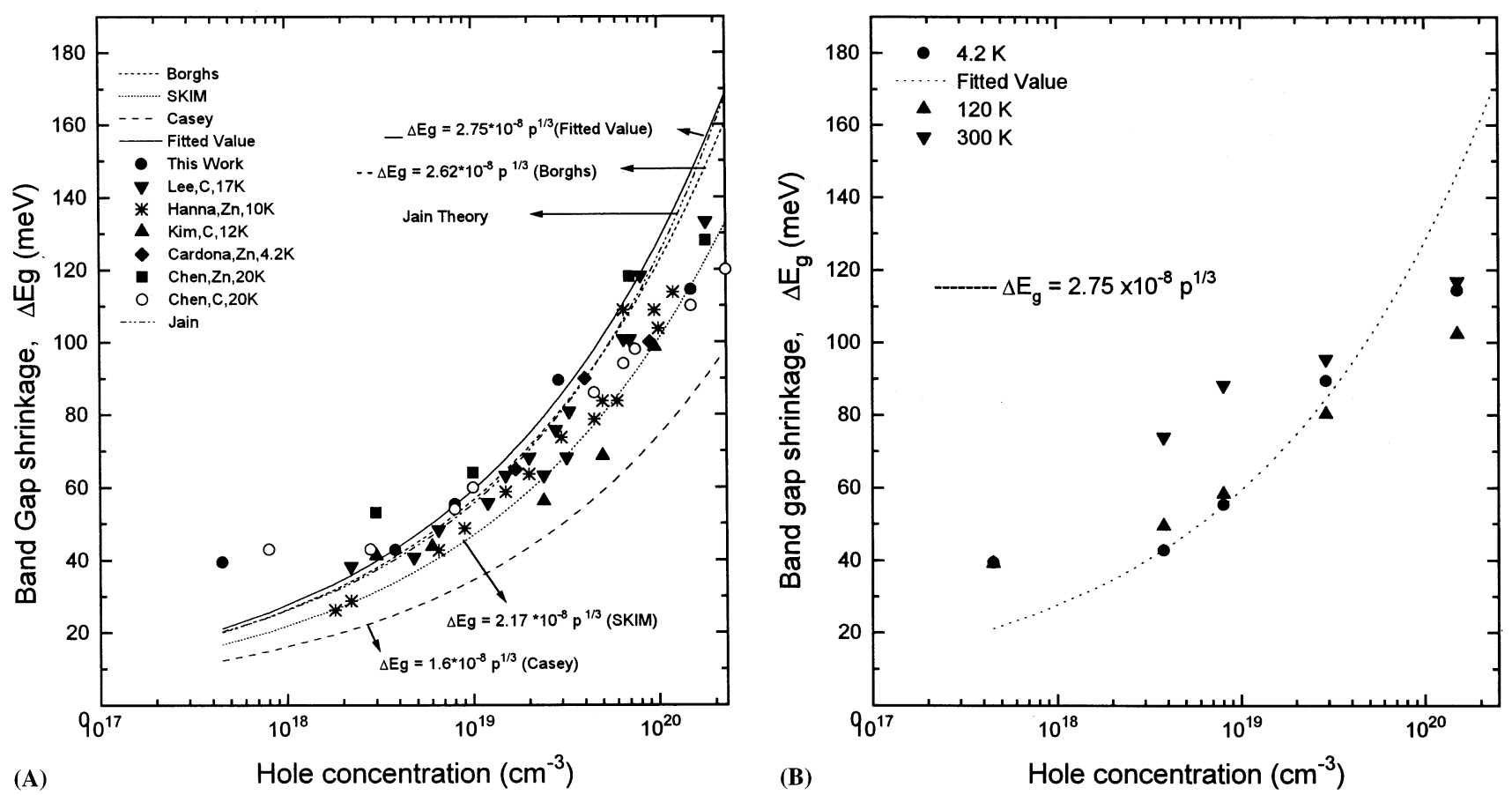

Fig. 10. (a). The band gap shrinkage of $\mathrm{Zn}$-doped GaAs epilayers as a function of hole concentration. (b). The band gap shrinkage of $\mathrm{Zn}$-doped GaAs epilayers as a function of hole concentration with different PL measurement temperatures.

tively, where $\mathrm{p}$ is the hole concentration in $\mathrm{cm}^{-3}$ and $\Delta E_{\mathrm{g}}(\mathrm{p})$ in $\mathrm{eV}$. The band gap shrinkage result also plotted in Fig. 10 (a) given by Casey and Stern [39]. These relations are considered to provide a useful tool for determination of hole concentration in $\mathrm{Zn}$-doped GaAs by low temperature PL measurement. From the Fig. 10 (b), we found that the band gap shrinkage or band gap narrowing of $\mathrm{Zn}$-doped GaAs measured at 4.2 and $120 \mathrm{~K}$ by PL measurement in the hole concentration range of $10^{17}-1.5 \times 10^{20} \mathrm{~cm}^{-3}$ are almost same. But the BGN at $300 \mathrm{~K}$ is higher compared to lower temperature PL measurement, which is expected since the peak energy shifted to lower energy with the PL measurement temperature. The decrease in $E_{\mathrm{p}}$ with the increase in temperature can be thought as the increase in hole density, since the PL peak energy shifted to lower energy side and hence the higher BGN at the higher temperature. It is found in Fig. 10 (a) that the BGNs are quite good for densities up to around $4 \times 10^{19} \mathrm{~cm}^{-3}$ of different dopants in the MOCVD growth process. For higher densities there are some deviations. These could be due to effects from self-compensation or acceptor clustering. Both these effects, which are expected to occur to higher degree with increasing doping density, would reduce the ion-contribution to the BGN [40].

\subsubsection{Effect of TMGa mole fraction on PL spectra}

To observe the effect of TMGa mole fractions on the luminescence properties of $\mathrm{Zn}$-doped GaAs, the PL measurement were carried out at $4.2 \mathrm{~K}$ specifically on those samples grown at different TMGa flow rates. Fig. 11 shows the PL spectra of Zn-doped GaAs for fixed $\mathrm{AsH}_{3}, \mathrm{DMZn}$ mole fractions. The three curves represent different TMGa flow rates. The hole concentrations increase with increasing TMGa mole fractions

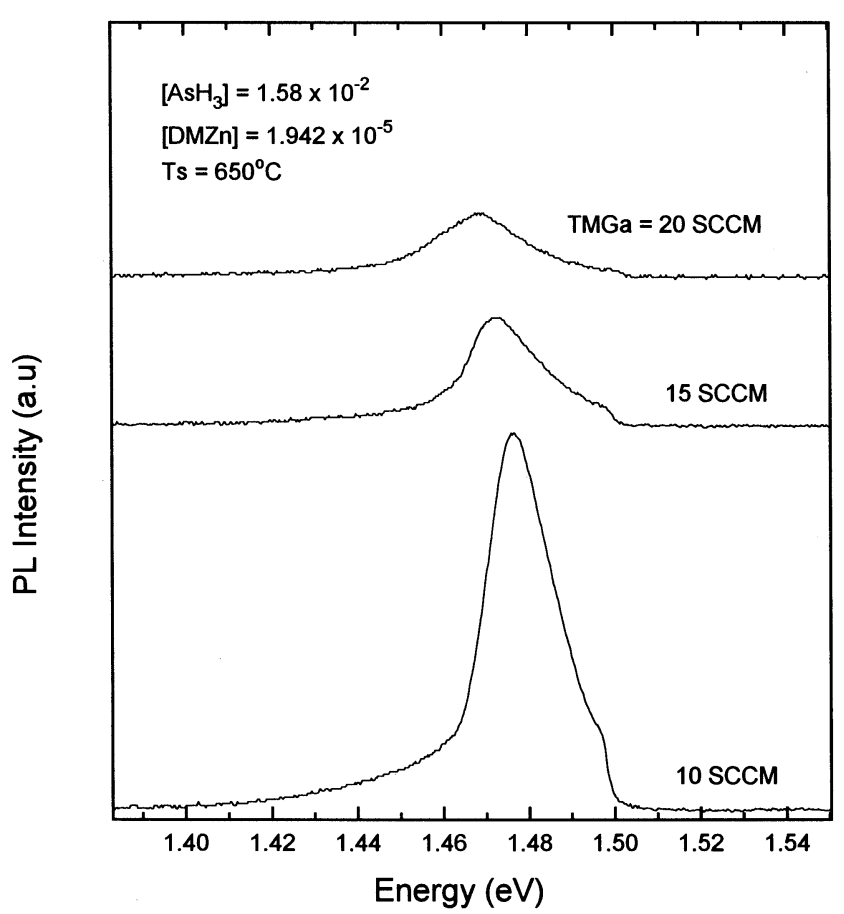

Fig. 11. 4.2 K PL spectra of Zn-doped GaAs epilayers as a function of TMGa flow rate. 
and the main peak is shifted to lower energy with increasing TMGa mole fractions. This can be explained as, for a fixed growth temperature and mole fraction of DMZn, the hole concentration increases linearly with increasing growth rate [8]. At high growth rates, the deposited zinc is absorbed into the epilayer before it can be evaporated from the surface. At lower growth rates, however, the $\mathrm{Zn}$ atoms diffuse to the surface, where they can evaporate. Thus, as the growth rate is increased, the residence time of $\mathrm{Zn}$ required for incorporation decreases, leading to increased $\mathrm{Zn}$ concentration via reduced $\mathrm{Zn}$ re-evaporation. Therefore, the increase in hole concentration with increasing TMGa partial pressure, the PL peak shifted to lower energy with TMGa and hence higher band gap narrowing.

\section{Conclusions}

Zn-doped GaAs epitaxial layers grown by low pressure metal organic chemical vapor deposition technique in the hole concentration range $1 \times 10^{17}$ to $1.5 \times 10^{20}$ $\mathrm{cm}^{-3}$ have been investigated by low temperature photoluminescence as a function of hole concentration and PL measurement temperatures, between 4.2 and $300 \mathrm{~K}$. From the PL spectra we have obtained an empirical relation for FWHM, peak energy and band gap shrinkage as a function of hole concentration. These relations are considered to provide a useful tool for determining the hole concentration by low temperature PL measurement. The donor-to-acceptor (e-A) peak shifts to low energy side as the hole concentration increases. The (e-A) peak shifts to lower energy side as the TMGa mole fraction increases due to the lowering of $\mathrm{Zn}$ residence time. The surface morphology of $\mathrm{Zn}$-doped GaAs is strongly dependent on the substrate surfaces below $600^{\circ} \mathrm{C}$. The growth temperature affects the $\mathrm{Zn}$ incorporation efficiency and a mirror smooth surface of having hole concentration $1.5 \times 10^{20} \mathrm{~cm}^{-3}$ at $600^{\circ} \mathrm{C}$ was obtained.

\section{Acknowledgements}

The authors wish to thank Bharat Electronics and ISRO for financial support in carrying out this work

\section{References}

[1] H.M. Manasevit, W.I. Simpson, J. Electrochem. Soc. 116 (1969) 1725.

[2] G.B. Stringfellow, Organometallic Vapour-Phase Epitaxy: Theory and Practice, Academic, San Diego, 1989.

[3] C.R Absernathy, F. Ren, P.W. Wisk, S.J. Pearton, R Esagui, Appl. Phys. Lett. 61 (1992) 1092.
[4] L.J. Guido, G.S. Jackson, D.C. Hall, W.E. Plano, N. Hollonayak, Jr, Appl Phys. Lett. 52 (1988) 522.

[5] J.A. Silberman, T.J. de LyonJ.M. Woodall, Appl. Phys. Lett. 58 (1991) 2126.

[6] E.F. Schubert, Doping in III-V Semiconductors, Cambridge University Press, Cambridge, 1993.

[7] P.J. Wang, T.F. Kuech, M.A. Tischler, P.M. Mooney, G. Scilla, F. Cardone, J. Appl. Phys. 64 (1988) 4975.

[8] S.Z. Sun, E.A. Armour, K. Zheng, C.F. Schaus, J. Cryst. Growth 113 (1993) 103.

[9] D. Olego, M. Cardona, Phys. Rev. B. 22 (1980) 886.

[10] H.D. Chen, M.S. Feng, P.A. Chen, K.C. Lin, J.W. Wu, Jpn. J. Appl. Phys. Part 1. 33 (1994) 1920.

[11] L.W. Yang, P.D. Wright, V. Eu, Z.H. Lu, A. Majerfeld, J. Appl. Phys. 72 (1992) 2063.

[12] M.C. Hanna, Z.H. Lu, A. Majerfeld, Appl Phys. Lett. 58 (1991) 164.

[13] B.J. Aitchison, N.M. Haegel, C.R. Abernathy, S.J. Pearton, Appl. Phys. Lett. 56, (1990) 1154.

[14] H.D. Chen, M.S. Feng, P.A. Chen, K.C. Lin, J.W. Wu, J. Appl. Phys. 75 (1994) 2210.

[15] L.P. Chen, C.Y. Chang, C.H. Wu, J. Appl. Phys. 61 (1987) 442.

[16] C.Y. Chang, L.P. Chen, C.H. Wu, J. Appl. Phys. 61 (1987) 1860.

[17] Y.K. Su, C.Y. Chang, T.S. Wu, Y.C. Chou, C.Y. Nee, J. Crystal Growth 67 (1984) 472.

[18] J.S. Lee, I. Kim, B.D. Choe, W.G. Jeong, Y.K. Sin, W.S. Min, J. Appl. Phys. 79 (1996) 9278.

[19] P.R. Hageman, M.H.J.M. deCroon, X. Tang, L.J. Giling, J. Crystal Growth 129 (1993) 281.

[20] K. Saito, T. Yamada, T. Akatsuka, T. Fukamachi, E. Tokumitsu, M. Konagai, K. Takahashi, Jpn. J. Appl. Phys. 28 (1989) 2081.

[21] D.A. Cusano, Appl. Phys. Lett. 7 (1965) 151.

[22] J.I. Pankove, J. Appl. Phys. 39 (1968) 5368.

[23] A.N. Titkov, E.I. Chaikina, E.M. Komova, N.G Ermakova, Sov. Phys. Semicond. 15 (1981) 198.

[24] G. Borghs, K Bhattacharayya, K. Deneffe, P. Van Mieghen, R. Mertens, J. Appl. Phys. 66 (1989) 4381.

[25] Z.H. Lu, M.C. Hanna, A. Majerfeld, Appl Phys. Lett. 64 (1994) 88.

[26] N.H. Ky, L. Pavesi, D. Araujo, J.D. Ganiere, F.H. Reinhart, J. Appl. Phys. 69 (1991) 7585.

[27] M.K. Hudait, P. Modak, S.B. Krupanidhi, Mat. Res. Soc. Symp. Proc. 421 (1996) 281.

[28] S.I. Kim, M.S. Kim, S.K. Kim, C. Lee, J. Appl. Phys. 74 (1993) 6128.

[29] M.K. Hudait, P. Modak, S. Hardikar, S.B. Krupanidhi, Solid State Commun. 103 (1997) 411.

[30] R.W. Glew, J. Cryst. Growth 68 (1984) 44.

[31] S.J. Bass, P.E. Oliver in: GaAs and related Compounds 1976, Inst. Phys. Conf. Ser. 33b, Inst. Phys., London, 1977 p.1.

[32] M.K. Hudait, P. Modak, S. Hardikar, S.B. Krupanidhi, J. Appl. Phys. 82 (1997) 4931.

[33] J.A.V. Vechten, J. Electrochem. Soc. 122 (1975) 419.

[34] J.A.V. Vechten, J. Electrochem. Soc. 122 (1975) 423.

[35] D.W. Shaw, J. Cryst. Growth 31 (1975) 130.

[36] B.E. Sernelius, Phys. Rev. B 34 (1986) 5610.

[37] S.I. Kim, M.S. Kim, Y. Kim, K.S. Eom, S.K. Min, C. Lee, J. Appl. Phys. 73 (1993) 4703.

[38] S.C. Jain, J.M. McGregor, D.J. Roulston, J. Appl. Phys. 68 (1990) 3747.

[39] H.C. Casey Jr., F. Stern, J. Appl. Phys. 47 (1976) 631.

[40] B.E. Sernelius in: GaAs and related Compounds 1987, Inst. Phys. Conf. Ser. 91 Inst. Phys., Bristol and Philadelphia, 1987 p.153. 